\title{
$\pi$ Scope: python based scientific workbench with MDSplus data visualization tool
}

\author{
S. Shiraiwa, T. Fredian, J. Hillairet, and J. Stillerman \\ Plasma Science Fusion Center, MIT, Cambridge, MA, 02139, USA \\ CEA, IRFM, F-13108 Saint Paul-lez-Durance, France
}

\begin{abstract}
A newly developed python based scientific data analysis and visualization tool, $\pi$ Scope (http://piscope.psfc.mit.edu), is reported. The primary motivation is 1) to provide an updated tool to browse the MDSplus data beyond existing dwscope/jScope and 2) to realize a universal foundation to construct interface tools to perform computer modeling from experimental data. To visualize MDSplus data, $\pi$ Scope has many features including overplotting different signals and discharges, generating various plot types (line, contour, image, etc.), performing in-panel data analysis using python scripts, and producing publication quality graphics. The logic to generate multi-panel plots is designed to be backward compatible with dwscope, enabling smooth migration for users. $\pi$ Scope uses multi-threading in data loading, and is easy to modify and expand due to its object-oriented design. Furthermore, A user can access the data structure both from a GUI and a script, enabling relatively complex data analysis workflow built quickly on $\pi$ Scope.
\end{abstract}

Keyword: MDSplus; wxPython; Matplotlib; python

\section{Motivation and introduction to $\pi$ Scope [1]}

MDSplus[2,3] is a de-facto standard of data acquisition and storage system in fusion research community. MDSplus comes with two visualization applications, dwscope[4] and jScope. These lightweight applications are sufficient for quick waveform displays during machine operation. However, for more complicated visualization or data analysis, such as showing EFIT equilibria, applications have to be prepared separately, and commercial software such as IDL and Matlab is commonly used for this purpose. An inhouse developed tool often lacks features such as generation of publication quality graphics and users are left to write their own visualization/analysis programs even for making a simple image plots. MDSplus only provides interface functions to read/write data for this purpose.

Making a better bridge between data stored in the MDSplus system to more involved data analysis is our primary motivation. To this goal, we developed a new python based data analysis environment named $\pi$ Scope, in which a user can write python scripts for analyzing and visualizing data, and we implemented MDSplus data visualization tool (Scope) on $\pi$ Scope. This approach realizes a convenient data analysis work environment, in which a user can browse data, and immediately start more complicated data analysis using the data shown in Scope window.

In the following, we provide a general introduction to $\pi$ Scope in Sec. 2. Section 3 discusses MDSplus data viewing feature (Scope). We summarize this report in Sec. 4.

\section{2. $\pi$ Scope}

$\pi$ Scope is prepared as a standalone script development environment. It is equipped with a syntax-highlighting text editor, a debugger, a variable browser, and a python shell. These features are similar to what is seen in other existing integrated development environments (IDE). However, $\pi$ Scope focuses more on data analysis rather than software development. In $\pi$ Scope, user data and analysis scripts (*.py) are managed in a project tree. As its name implies, the project has a tree structure and all objects in the tree can be accessed by connecting object's names by $\operatorname{dot}($.). Built-in tree browser provides a convenient way to access to an object in the project tree.

The tree is a data structure on memory but at the same time, $\pi$ Scope automatically constructs a directory structure corresponding to the tree. $\pi$ Scope uses this disk space to keep a data file associated to a tree object. A user can access the data file via a data object. $\pi$ Scope comes with various data objects to support a variety of data files including both generic data formats, such as netCDF, fortran name list, .mat (MATLAB) file, and .sav (IDL) file, and fusion specific data formats, such as EFIT g-file. Data objects are designed as an add-on module. A user can write new add-on to support a new file/data format easily. $\pi$ Scope is equipped with a $2 \mathrm{D}$ plotting figure window. We uses wxPython[5] for the GUI toolkit and Matplotlib[6] for drawing 2D graphics. Although Matplotlib is used as an underlining drawing library, the figure window 
in $\pi$ Scope is more powerful than what is available in Matplotlib. We developed a variety of GUI elements so that a user can edit axis range, panel layout, symbol colors, and annotate graphics by legend, embedding image and so on. User's editing is undo/redo-able and final results can be saved in various graphics formats, such as eps, pdf, svg, png. However, since we need to wrap Matplotlib graphics object by the tree objects in $\pi$ Scope, not all of plotting types in Matplotlib is available in $\pi$ Scope. Types of plot currently supported is shown in Fig.1

In $\pi$ Scope, the figure window is also data in the project tree, which is called a book object. The book object can have multiple page objects and each page object can have multiple axes objects, each of which represents a set of $\mathrm{x}, \mathrm{y}, \mathrm{z}$ and color bar axes. Then, each axes object can have various plot objects. These objects know how to draw Matplotlib graphics
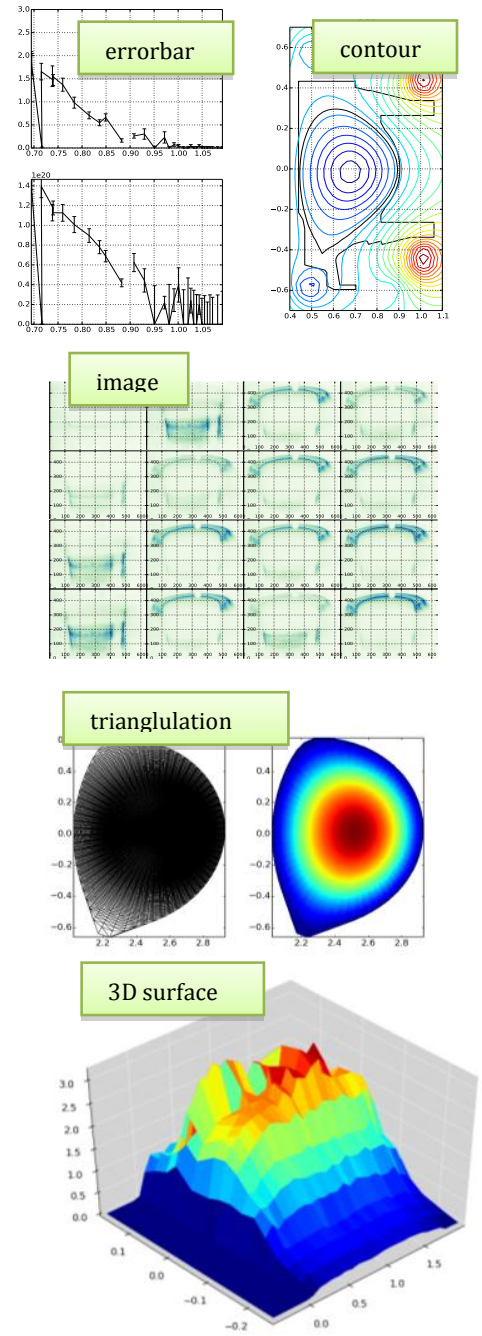

Fig. 1: Plot types supported in $\pi$ Scope. These graphics are made by directly taking the screen capture of figure generated using data from MDSplus server. on $\pi$ Scope's canvas panel (a subclass of wx.Panel) and are used as building blocks to implement Scope for MDSplus.

\section{MDSplus data visualization tool (Scope) in $\pi$ Scope}

MDSplus data visualization tool in $\pi$ Scope is called Scope (Fig 2). Scope inherits a look and feel from dwscope and jScope. A window is split into multiple subplots, which are called "panels", where various signals are shown. However, Scope in $\pi$ Scope provides many additional features to visualize MDSplus data, which are unavailable in either dwscope or jScope.

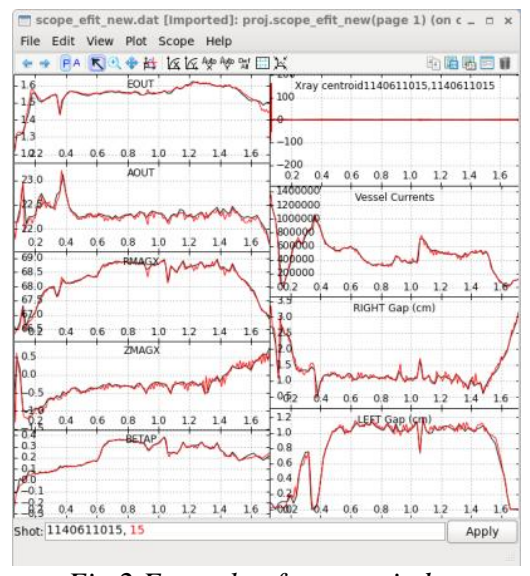

Fig 2 Example of scope window

\section{1 plotting}

Scope allows various plot types (line, contour, image, etc.) shown in Fig. 1. Since Scope constructs a tree of figure objects in the project tree by itself, once a data is read from the MDSplus server, a user can access all the data displayed in the scope window.

Over-plotting is one of features largely enhanced in the scope. A user can over-plot different discharges or different signals in the same panel or among multiple pages. In the multi-page mode, Scope automatically generates a sequence of figure pages formatted in the same way and generates graphics. Typical usage of this mode includes showing a time series of 2D data (such as camera images or plasma equilibria)

\subsection{In-panel data analysis}

Scope supports in-panel data analysis. A user can use either TDI or python for in-panel analysis, which can be setup in a signal setup window shown in Fig. 3. TDI based in-panel data analysis is enhanced from what is available in dwscope. A user can define unlimited number of variables using TDI, not limited to " $x$ " and " $y$ ". Python based in-panel analysis is new in Scope and is performed after 


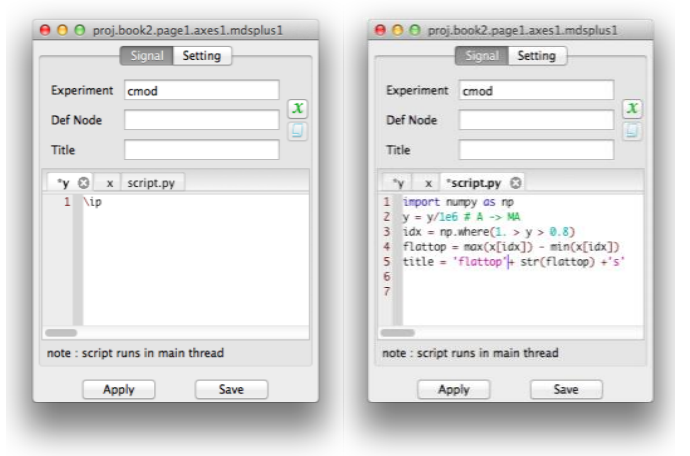

Fig. 3: Signal setting window. Panels for in-panel data analysis using TDI (left) and python (right) allows for processing raw data in two languages.

TDI based in-panel analysis. Scope evaluates TDI expressions and set results in a python dictionary, which is then passed to a python script. Output of the python scripts is a set of variables, whose names are pre-defined and are used to generate graphics.

3.4 Publication quality graphics generation. Generating publication quality graphics directory from the Scope window graphics is one of features, in which we devoted significant effort. A user can simply collect curves from a Scope window by cut-and-paste to a figure window and then edit it for the final publication production.

Since Scope runs on $\pi$ Scope, a user can use $\pi$ Scope's convenient figure editing features in Scope. A user can edit axis range, panel layout, symbol colors, and annotate graphics by legend, embedding image and so on. User's editing is undo/redo-able. Final results can be either saved in various graphics formats, such as eps, pdf, svg, png and so on, stored in OSwide clipboard, or sent as an E-mail attachment using built-in E-mail post office.

\subsection{Easy migration from dwscope}

In order to minimize an effort required when migrating from dwscope, the logic to produce multi-panel plots is designed to be backward compatible with dwscope. Furthermore, Scope supports importing an existing dwscope file. Scope extracts information such as (but not limited to) panel layout, signal TDI expressions, line style and symbol, global default settings, panel position in window, and variables from a dwscope file, and sets up a Scope window correspondingly. By importing a dwscope file in Scope, a user can immediately benefit from various additional features such as overplotting. The importer also supports global TDI expressions, which is used to set segment variable to read a segmented data from a long pulse (or continuous) data.
Previously, only way to open two scope screens is to launch two scope applications. In $\pi$ Scope, a user can open multiple Scope windows and import different dwscope files in each Scope. This way, a user can manage set of scope files as a single project.

Presently importing jScope file is not yet supported. However, providing a similar level of support to dwscope is not difficult.

\subsection{Object oriented design}

Object oriented design is employed throughout $\pi$ Scope. Scope (MDSScope object) is a subclass of wx.Frame class object, and a user can easily expand it by subclassing Scope class. For example, a developer can easily add a custom action after loading data such as processing a time trace signals and convert them to dynamic spectrum,

Furthermore, the engine that loads data from the MDSplus server and processes data using python scripts is implemented as a separate class object. Therefore, a user can write the application that has a user interface completely different from Scope. Indeed, $\pi$ Scope comes with "no window Scope", which takes the same book object, and loads the data using the engine, but instead of showing the data on screen, it only stores data in the project tree.

\subsection{Multithreading and multiprocessing}

The engine to load data and to perform inpanel data processing employs a multithreaded design, consisting from two control threads and many workers. Workers run either in different threads (threading module) or in different processes (multiprocessing module).

Using this engine, Scope supports parallel processing of data loading and in-panel analysis in three different modes: panel level parallelism, discharge level parallelism, and page level parallel. In panel level parallelism, all the sub-panels are processed in parallel. In discharge level parallelism, signals for different discharges are processed in parallel. In page level parallelism, different pages are processed in parallel.

We considered two scenarios in which loading data becomes slow. One is due to the latency of network. This could be an issue especially when the MDSplus server is physically located in a long distance away. In this case, using multithreading workers is better practice. The other scenario is when data processing by a python script (in-panel analysis) is the bottleneck. In such a case, multiprocessing based parallelism allows for taking advantages of multiple CPU cores and tends to be a better choice, although the result of analysis needs to be transferred to the main 
python process via multiprocess.queue module.

In the Scope engine, the worker thread and the MDSplus event listener threads are the only components that directly depend on MDSplus. Supporting other database accesses can be realized relatively easily by replacing these two components.

\subsection{Scriptable Scope}

The Scope object is a standard python object and has pre-defined methods to support data analysis by a python script, including opening a book object and loading a particular discharge. The book object can be an object in the project tree, or a user can tell Scope to import a dwscope file. Once data is read, since Scope stores signals in memory, a user can reuse the data in the following analyses.

Fig. 4 illustrates the convenience realized by this design. In this example, a python script shown in Fig. 4 (2) imports a dwscope file to read a brightness signal of soft X-ray detector on Alcator C-Mod and compute a dynamic spectrum of the signal. Note that after importing dwscope and reading the data from server (line \#4-6), the script retrieves the data from a timetrace object in line \#15. In line $\# 15$, v1 is the scope window, which has an attribute named book. The data read from MDSplus server is represented as a timetrace

(1)

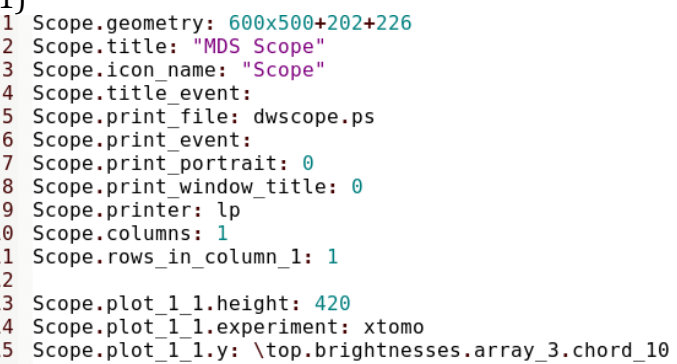
signal on C-Mod (this file is saved as " $\sim /$ test.dat" and opend in the script) (2) A python script to open the dwscope file shown in (1), to load a discharge, and to show a dynamic spectrum of signal. object, which is accessed using chain of object attribute access. The export method of the timetrace object returns the data as python dictionary array. After obtaining data in line $\# 15$, the script opens a new window and show a dynamic spectrum in line \#17-\#20. Thanks to underlining matplotlib graphics, these steps are similar to matlab commands lowering a initial learning curve for a new user. Also, notice that the script does not call python MDSplus object methods directly, since the Scope engine handles such a low-level layer.

\section{Summary}

$\pi$ Scope is a newly developed python based scientific data analysis and visualization tool. It provides feature rich data browser stored in MDSplus data system. In this report, we discussed our high level goal of developing new software and introduced features realized in $\pi$ Scope.

One of goal of $\pi$ Scope development is to bridge smoothly between experimental data and computer modeling. $\pi$ Scope is equipped with pre-defined objects to support constructing data analysis workflow in project, such as Parameter, Data, Script, Model, and Solver. Using those objects, a simulation workflow to perform MSE constrained EFIT and to evaluate lower hybrid current drive using the GENRAY/CQL3D code package was built. This workflow management feature will be discussed in detail in a separate publication.

$\pi$ Scope has been tested extensively during FY14 run campaign of Alcator C-Mod both for machine operation and post-discharge data analysis/computer modeling. $\pi$ Scope has also been tested with Tore Supra and JET database using their MDSplus database wrapper. $\pi$ Scope is distributed under GNU v3 license, developed for multiple platforms including Linux, Windows and MacOSX and available from $\pi$ Scope website [1].

Acknowledgement:

This work supported by US Department of Energy cooperative agreement DE-FC02-99ER54512.

Reference:

[1] $\pi$ Scope wiki (http://piscope.psfc.mit.edu)

[2] Stillerman, J.A., Fredian, T.W., Klare, K.A.,

Manduchi, G., "MDSplus data acquisition system", Review of Scientific Instruments, January 1997 68(1) pp 939-942.

[3] http://www.mdsplus.org

[4] Fredian,T.W., Stillerman, J.A., "X-Window based user interface for data acquisition and display", Review of Scientific Instruments Vol 61 No 10 October 1990 pp 3283-3285.

[5] http://www.wxpython.org [6] http://www.matplotlib.org 